\title{
Gyrus Rectus Cavernoma Masquerading as an Intracranial Aneurysm: A Case Report and Review of Literature
}

\author{
Zahraa M Kareem ${ }^{1}$, Mohamed M Arnaout ${ }^{2}$, Ruqayah A Al-Baidar ${ }^{1}$, Zahraa F Al-Sharshahi ${ }^{3 *}$ and Samer S \\ $\mathrm{Hoz}^{3}$ \\ ${ }^{1}$ Medical Student, College of Medicine, University of Baghdad, Baghdad, Iraq \\ ${ }^{2}$ Department of Neurosurgery, Faculty of Medicine, Zagazig University, Zagazig/Sharqia, Egypt \\ ${ }^{3}$ Department of Neurosurgery, Neurosurgery Teaching Hospital, Baghdad, Iraq
}

*Corresponding author: Zahraa Al-Sharshahi, MB, BCh BAO (NUI, RCSI), LRCP \& SI, Department of Neurosurgery, Neurosurgery Teaching Hospital, Baghdad, Iraq, Tel: 009647826219848

\begin{abstract}
Introduction: A cerebral cavernous malformation [CCM] is a cluster of abnormally thin and dilated blood vessels. In rare instances, diagnosis can be clouded by the possibility of an intracranial aneurysm. In this paper, we report a case of CCM that was initially misdiagnosed as intracranial aneurysm. We conduct a review of the literature on similar cases.
\end{abstract}

Case presentation: A healthy, 43-year-old man had a three-month history of recurrent headaches and seizure episodes. Imaging studies, including CT, GRE T2W MRI, and CTA were inconclusive. The case was surgically handled; a gyrus rectus CCM, but no aneurysm, was found and excised totally.

Methods: A PubMed Medline database search was conducted using the following medical subject headings [MESH] terms and keywords: "cavernous hemangioma" OR "cavernous angioma" OR "cavernous malformation" OR "CCM" AND "intracranial aneurysms" OR "aneurysm". The focus of the review was to identify cases where an aneurysm was initially misdiagnosed as a CCM and vice versa.

Results: A total of 15 studies [16 cases] were included in the review. Of those, there were eight studies that described a CCM mimicking an intracranial aneurysm. CCMs that mimicked aneurysms were mostly located at the cranial nerves $[n=3 / 9]$.

Conclusion: CCMs and intracranial aneurysms may, in rare instances, present with equivocal clinical and radiological features. In such cases, detailed radiological examination is necessary to distinguish between the two lesions, using radiological signs and the presence/absence of intracranial bleeding as diagnostic clues.

\section{Keywords}

Cerebral cavernous malformations, Microsurgical resection, Intracranial aneurysms

\begin{abstract}
Abbreviations
CCM: Cerebral Cavernous Malformation; ACA: Anterior Cerebral Artery; Acom: Anterior Communicating Artery; AVM: Arteriovenous Malformation; CT: Computed Tomography; CTA: Computed Tomography Angiography; DSA: Digital Subtraction Angiography; GRE: Gradient Recalled Echo; MRI: Magnetic Resonance Imaging; MRA: Magnetic Resonance Angiography; ICA: Internal Carotid Artery; ICH: Intracerebral Hemorrhage; IVH: Interventricular Hemorrhage; MCA: Middle Cerebral Artery; Pcom: Posterior Communicating Artery; PICA: Posterior Inferior Cerebellar Artery; SAH: Subarachnoid Hemorrhage; T1WI: T1-Weighted Image; T2WI: T2-Weighted Image; A1: PreCommunicating Segment Of Anterior Cerebral Artery; A2I: Post-Communicating Segment Of Anterior Cerebral Artery
\end{abstract}

\section{Introduction}

A CCM is a cluster of abnormally thin and dilated blood vessels located in the brain or parenchyma. They are also known as cerebral cavernous malformations or cavernous hemangiomas [1]. CCMs represent about $10 \%-15 \%$ of all central nervous system vascular malformations and are mostly supratentorial [2]. They can present at any age, with seizures being the most common presenting symptom. However, they can also be identified incidentally on magnetic resonance imaging [MRI] ordered for other unrelated complaints

Citation: Kareem ZM, Arnaout MM, Al-Baidar RA, Al-Sharshahi ZF, Hoz SS (2021) Gyrus Rectus Cavernoma Masquerading as an Intracranial Aneurysm: A Case Report and Review of Literature. Neurosurg Cases Rev 4:085. doi.org/10.23937/2643-4474/1710085

Accepted: October 09, 2021; Published: October 11, 2021

Copyright: (C) 2021 Kareem ZM, et al. This is an open-access article distributed under the terms of the Creative Commons Attribution License, which permits unrestricted use, distribution, and reproduction in any medium, provided the original author and source are credited. 


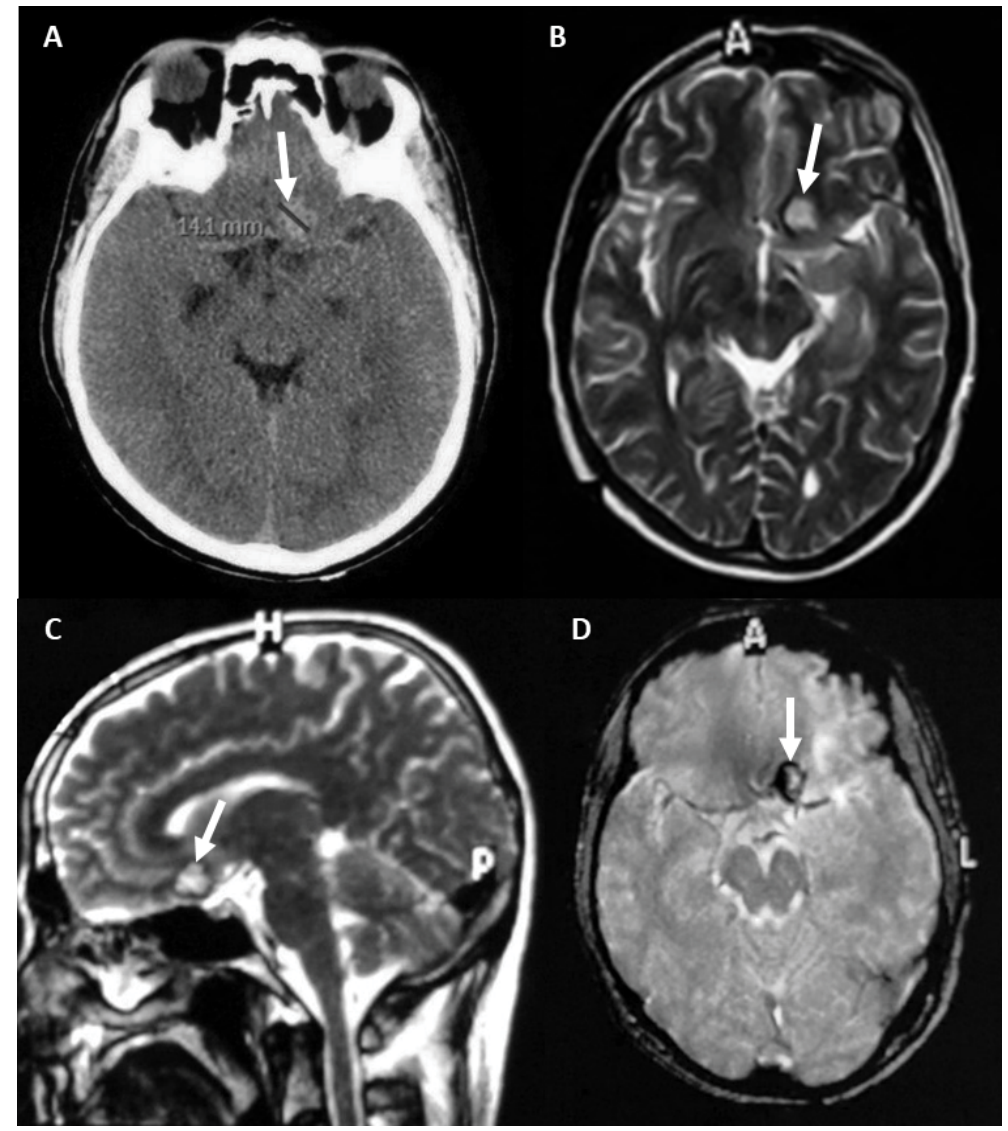

Figure 1: A) Non-contrast axial section brain CT scan showing a small relatively round hyperdense lesion [arrow] in the posterior part of left gyrus rectus without calcification or perilesional edema. No SAH is seen; B,C) Axial and sagittal sections T2-Weighted; D) GRE MRI images demonstrate a relatively small hyperintense lesion [arrow] surrounded by hypointense rim in the posterior part of gyrus rectus.

CT: Computed Tomography; SAH: Subarachnoid Hemorrhage; GRE: Gradient Recalled Echo; MRI: Magnetic Resonance Imaging.

[3]. A co-existing developmental venous anomaly [DVA] is nearly found in one-third of all CCM cases, and identification of such anomalies is essential for surgical management $[4,5]$.

Furthermore, symptomatic cases are at a higher risk for hemorrhaging compared to the asymptomatic ones, which carry a less than $1 \%$ bleeding risk [6]. Hemorrhagic CCM may take several radiological pictures, even mimicking malignant tumors $[7,8]$. The uncertainty between intracranial aneurysms and cerebral cavernous malformations is rarely described in the literature $[9,10]$. Herein, we report a case of CCM mimicking intracranial aneurysm along with its diagnostic and management pathway. Besides, we present a literature review of CCMs mimicking intracranial aneurysms and vice-versa.

\section{Case Presentation}

A previously healthy, 43-year-old man presented with a three-month history of recurrent frontal headache and seizure episodes. The seizures were absence in nature and were not controlled by Carbamazepine. On examination, the patient was neurologically intact. The initial brain computed tomography [CT] scan showed a relatively small, rounded, hyper-dense lesion [14 $\mathrm{mm} \times 8$ $\mathrm{mm}$ ] in the posterior medial part of the gyrus rectus just close to the ACA complex in the interhemispheric fissure. No calcification, perilesional edema, or subarachnoid hemorrhage $[\mathrm{SAH}]$ were detected. The initial suspicion was that of an unruptured ACA aneurysm (Figure 1A). We opted to perform a brain magnetic resonance imaging [MRI] as catheter angiography is not available at our institute. The brain MRI showed a hyperintense lesion on T1- and T2-weighted images, surrounded by a hypointense rim. The gradient recalled echo [GRE] sequence showed a bloomy dark appearance of the lesion. The cerebral CT angiography [CTA] revealed no trace of an intracranial aneurysm. However, the probability of a thrombosed aneurysm could not be completely excluded. The decision was made to diagnose and treat the lesion surgically, using the pterional subfrontal approach. Our surgical strategy was first to dissect the ACA, searching for aneurysm, and if it were not there, then the posterior gyrus rectus would be dissected to excise the CCM.Following ACA dissection, which revealed no abnormality, the gyrus rectus CCM was identified, dissected and removed (Figure 2). The histopathological examination confirmed the diagnosis of CCM. There were no surgical complications, and the postoperative recovery was uneventful. The patient was discharged on post-operative day seven. He was 


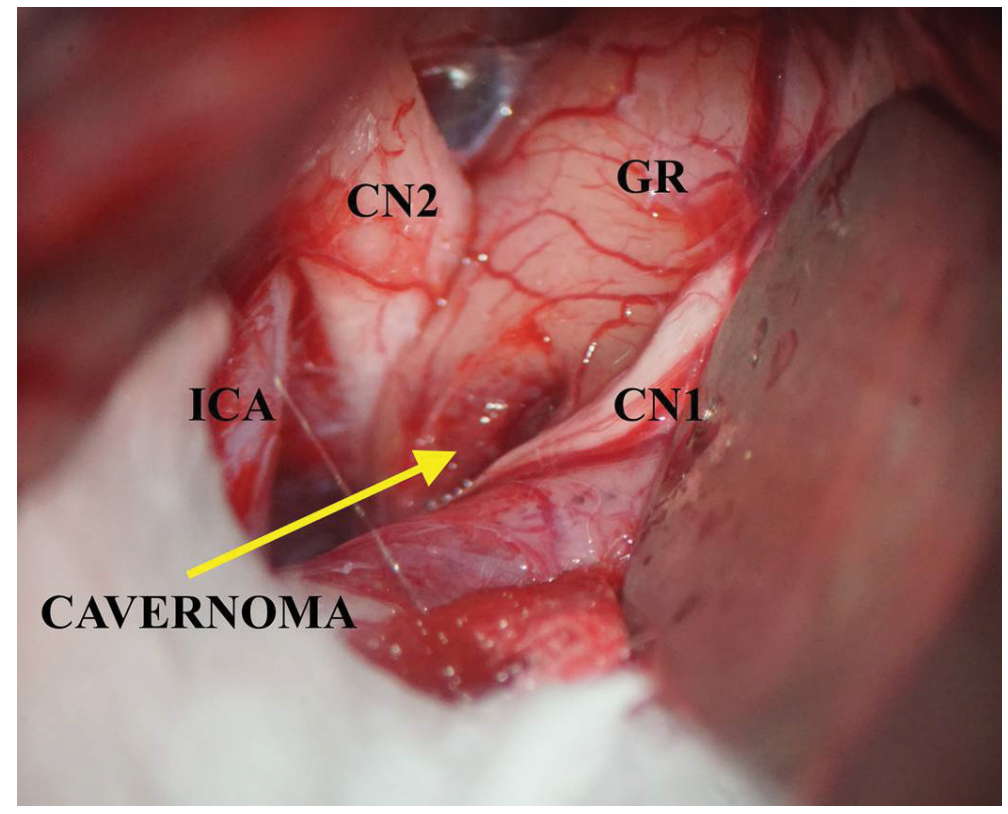

Figure 2: Intraoperative microscopic images visualizing the left posterior gyrus rectus CCM. ICA: Internal Carotid Artery; GR: Gyrus Rectus; CN1: Cranial Nerve I; CN2: Cranial Nerve II

advised to continue on the anti-epileptic drugs for up to two years. A three-monthly follow-up for drug level monitoring was scheduled. On his six-month follow-up visit, the patient was neurologically intact, and seizurefree. Laboratory results were within normal limits and both the follow-up CT and MRI studies showed no remnants of the CCM.

\section{Patients and Methods}

\section{Literature review}

A PubMed Medline database search was conducted using the following combination of medical subject headings [MESH] terms and keywords:"cavernous hemangioma" OR "cavernous angioma" OR "cavernous malformation" OR "CCM" AND "intracranial aneurysm" OR "aneurysm". Only articles reporting human studies, and those published in peer-reviewed journals were included.

The focus of the review was to identify cases where an aneurysm was initially misdiagnosed as a CCM and vice versa. A total of 653 results were obtained from the initial scan. The abstract analysis was carried out by two independent reviewers. The reference list of each paper was then reviewed for further studies. The following set of data was collected from each study: Age, gender, presenting signs/symptoms, radiological findings, initial diagnosis, postoperative diagnosis and outcomes.

\section{Results}

A total of 15 studies [16 cases] were included in the review. Of those, there were eight studies [nine cases] [including the present case] that described a CCM mimicking an intracranial aneurysm (Table 1). The remaining seven studies [seven patients] described the opposite situation wherein an intracranial aneurysm was initially misdiagnosed as a CCM (Table 2).

In a total of 16 cases, 9 [56\%] were male and 7 [43\%] female. The age distribution was between 19 and 69 years, with an average age of 42 years and a median age of 44 years. Of the patients originally misdiagnosed with aneurysm, six patients complained of headache [2,3,58]. One patient had left-sided, mild hemiparesis and hypoesthesia [1]. Two patients had third cranial nerve palsy and only one patient had seizures. As for patients with intracranial aneurysm misdiagnosed as CCM, five patients had headache [11-15] and only two patients had seizures $[16,17]$.

In most cases, preoperative imaging studies did not show the traditional radiological properties of CCMs. In certain cases, it has also offered the appearance of an expansive, encapsulated, multi-cystic, vascular lesion [18]. In others, the MRI showed inconclusive findings suggesting partially or completely thrombosed aneurysms $[19,20]$. Interestingly, there were two cases of acute subarachnoid hemorrhage associated with CCM $[19,21]$, and two cases of intracranial hemorrhage with CCM $[22,23]$.

Aneurysms that mimicked CCMs were mostly located at the middle cerebral artery [MCA], [n=3]. The next most common location was the posterior inferior cerebellar artery [PICA], $[n=2]$. In the remaining two cases, the aneurysm was located at the anterior cerebral artery $[A C A]$, and the anterior communicating artery [Acom].

The locations of CCMs that mimicked aneurysms were as follows: the oculomotor nerve $[n=2]$, the optic nerve $[n=1]$, the cerebellum $[n=1]$, foramen magnum - intradural extramedullary [ $n=1]$, and the gyrus rectus $[n=1]$. 
In a total of 16 cases, $87 \%$ of patients were surgically handled, and $12 \%$ were conservatively controlled. Good outcomes have been reported in $94 \%$ of cases [ $n=15$ ]; only one case has had a poor outcome.

\section{Discussion}

Intracranial CCMs are common vascular malformations formed of abnormal clusters of hyalinized capillaries surrounded by hemosiderin deposits with a gliotic margin [13]. Clinically, CCMs can present with a wide range of symptoms, including occipital headache, hemiparesis, siezures, and hypoesthesia; these symptoms tend to occur in alternate cycles of exacerbation and recovery [24]. Compared to CCMs, intracranial aneurysms are bulged, weakened areas in the intimal layer of the arterial wall. Although most aneurysms present with signs and symptoms of SAH, some may have mass effects, particularly large and thrombosed aneurysms [25].

\section{Location of the lesion}

Approximately $70-80 \%$ of brain CCMs are found in the supratentorial region of the brain [26], whereas only $15 \%$ are found in the infratentorial part [27]. Brainstem CCMs make up 9-35\% of all CCMs in the central nervous system [14]. The majority of cerebral and spinal cord CCMs are intraparenchymal and only a limited number can be found in the epidural space [15].

The intradural extra-medullary position at the foramen magnum is an infrequent location for CCMs, and lesions at this site are more likely to be aneurysms or tumors [27]. CCMs have also been rarely found within the cranial nerves, particularly the optic nerve [28]. Distal internal carotid artery aneurysms [including posterior communicating artery aneurysms] could be misdiagnosed as cranial nerve CCMs due to the proximity of these two sites. This concept was highlighted by our review, which showed that the cranial nerves were the most common site for CCMs that mimicked cerebral aneurysms. Such a misdiagnosis becomes more common in the presence of a thrombosed aneurysm [20]. In our case, the CCM was located at the posterior part of gyrus rectus, which is an uncommon site for CCM formation, favoring the diagnosis of an ACA aneurysm over a CCM [10].

The study found that MCA was the most common source of aneurysms that mimicked CCMs; a result that can be partially explained by the vulnerability of such aneurysms to thrombosis, producing mass effects [9].

PICA was the second most frequently reported site for aneurysms that mimicked CCMs. This observation can be linked, in part, to the location of PICA. PICA aneurysms, especially those in the distal segment of the artery, can closely resemble cerebellar CCMs $[12,15]$.

\section{Radiological characteristics}

CCMs have the appearance of multi-lobulated, mulberry-like bulging lesions. Such abnormally-shaped vessels are prone to low-flow areas that may be calcified or restrain stagnated blood, leading to varying degrees of thrombosis or degradation $[24,26]$. CT scans have low sensitivity and specificity in diagnosing CCMs. High-field magnetic resonance imaging [MRI] is the best radiological modality for detecting these lesions [29].

The choice of the imaging modality for CCMs depends on the size, location, and duration of previous hemorrhagic episodes, if any. Normally, the non-enhanced CT scan reveals an isodense to mildly hyperdense lesion. Evidence of stippled calcifications is also seen in $60 \%$ of larger lesions [23]. The MRI findings are more accurate, demonstrating the appearance of the so-called "popcorn ball" with varying degrees of mixed hyper-and hypointensities in the T1 \& T2 weighted images. The MRI also shows a surrounding low-signal intensity of hemosiderin rim [30] angiomas are angiographically latent lesions; as such, the use of angiography can be deemed unnecessary in the evaluation of CCMs [31]. Angiography, however, remains important to diagnose giant thrombosed aneurysms that can mimic CCMs [32].

In the present case, the initial CT scan data suggested the diagnosis of an unruptured ACA aneurysm, while the MRI findings were pointing towards a CCM. Due to the unavailability of angiography at our institution, neither diagnosis could be ascertained pre-operatively.

\section{Intracranial bleeding patterns}

Intracranial CCMs have a $2.39 \%$ annual risk of intracranial bleeding [33]. This risk is up to 7.78 times higher in posterior fossa CCMs, particularly in cases of prior episode[s] of hemorrhage [34]. Recurrent bleeding can cause enlargement of the CCM with subsequent mass effects [27]. The non-enhancing heterogeneous "popcorn" appearance with the surrounding hemosiderin rim on the $\mathrm{CT}$ and MRI scans is attributed to recurrent bleeding episodes.

On the other hand, ruptured intracranial aneurysms commonly present with SAH. Different bleeding patterns, such as subdural hematoma or intraparenchymal hemorrhage are of a rare occurrence compared to the $\mathrm{SAH}$. Thus, CCMs and aneurysms may intersect in the patterns of intracranial bleeding, necessitating proper radiological evaluation [35].

Good outcomes have been reported in the majority of the cases $93.6 \%$ [ $n=15$ ], with only $6.4 \%$ of cases [ $=1 / 16$ ] having poor outcomes. Given the small number of the studies and their form [i.e., all included studies are case reports], the result might not be generalizable in all related cases. The theoretical probability of poor surgical outcomes remains high, particularly when 
considering the impact of such misdiagnoses on the surgical plan, approach, and technique.

This review underlines the difficulty of reaching a definitive diagnosis of certain vascular brain lesions. Neuroradiological imaging alone cannot provide an accurate provisional diagnosis in certain cases with ambiguous lesions, which are often identified intraoperatively.

One drawback of our analysis is that all the included studies were case reports, with a maximum of two cases per study. As a result, several questions remain unanswered concerning the optimal diagnostic and management strategies for CCMs masquerading as intracranial aneurysms and vice versa. Large, multicenter, prospective cohort studies are required before the obscurities surrounding these lesions can be elucidated.

\section{Conclusions}

CCMs and intracranial aneurysms are two distinct neurovascular pathologies, both of which have conclusive clinical and radiological features. In rare cases, however, one lesion can masquerade as the other, creating a diagnostic uncertainty. In such instances, a detailed radiological examination is necessary to distinguish between the two lesions, using radiological signs and the presence/absence of intracranial bleeding as diagnostic clues.

\section{Declarations}

\section{Ethics approval and consent to participate}

None-required.

\section{Consent for publication}

None-required.

\section{Availability of data and materials}

Yes.

\section{Competing interests}

Nothing to declare.

\section{Funding}

None-received.

\section{Acknowledgements}

None.

\section{Author Contributions}

Z.M.K: Literature review; M.M.A: Manuscript revision; R.A.A: Manuscript revision; Z.F.A: Manuscript drafting; S.S.H: Case Identification and management.

\section{References}

1. Steiger HJ, Tew JM (1984) Hemorrhage and epilepsy in cryptic cerebrovascular malformations. Arch Neurol 41: 722-724.
2. Washington CW, McCoy KE, Zipfel GJ (2010) Update on the natural history of cavernous malformations and factors predicting aggressive clinical presentation. Neurosurg Focus 29: E7.

3. Kivelev J, Niemela M, Kivisaari R, Hernesniemi J (2010) Intraventricular cerebral cavernomas: A series of 12 patients and review of the literature. J Neurosurg 112: 140149.

4. Batra S, Lin D, Recinos PF, Zhang J, Rigamonti D (2009) Cavernous malformations: Natural history, diagnosis and treatment. Nat Rev Neurol 5: 659.

5. Abdulrauf SI, Kaynar MY, Awad IA (1999) A comparison of the clinical profile of cavernous malformations with and without associated venous malformations. Neurosurgery 44: 41-46.

6. Zabramski JM, Wascher TM, Spetzler RF, Johnson B, Golfinos J, et al. (1994) The natural history of familial cavernous malformations: Results of an ongoing study. J Neurosurg 80: 422-432.

7. Gross BA, Lin N, Du R, Day AL (2011) The natural history of intracranial cavernous malformations. Neurosurg Focus 30: E24.

8. Shirvani M, Hajimirzabeigi A (2017) Intraventricular cavernous malformation: Review of the literature and report of three cases with neuroendoscopic resection. J Neurol Surg Part A Cent Eur Neurosurg 78: 269-280.

9. Hegde AN, Mohan S, Lim CCT (2012) CNS cavernous haemangioma: "Popcorn" in the brain and spinal cord. Clin Radiol 67: 380-388.

10. Lew SM, Morgan JN, Psaty E, Lefton DR, Allen JC, et al. (2006) Cumulative incidence of radiation-induced cavernomas in long-term survivors of medulloblastoma. $\mathrm{J}$ Neurosurg Pediatr 104: 103-107.

11. Farias JP, Trindade AM (1997) Giant distal anterior cerebral artery aneurysm not visualized on angiography: Case report. Surg Neurol 48: 348-351.

12. Lim D-H, Jung S, Jung T-Y, Kim T-S (2008) An unusual case of a thrombosed giant distal PICA aneurysm simulating a large cavernous angioma. J Korean Neurosurg Soc 43: 155.

13. Kumar VRR, Madhugiri VS, Sasidharan GM, Gundamaneni SK, Yadav AK, et al. (2015) Totally thrombosed giant anterior communicating artery aneurysm. J Neurosci Rural Pract 6: 245-247.

14. Trungu S, Bruzzaniti P, Forcato S, Cimatti M, Raco A (2017) Completely thrombosed distal middle cerebral artery aneurysm mimicking a cavernous angioma: Case report and review of the literature. World Neurosurg 103: 955-e1.

15. Zhang H, Deng G, Liu B, Wang J, Wang L, et al. (2019) Thrombotic aneurysm of posterior inferior cerebellar artery. World Neurosurg 129: 526-530.

16. Oka H, Kurata A, Miyasaka Y, Kobayashi I, Oomomo T, et al. (1994) Completely thrombosed large aneurysm of the distal middle cerebral artery: A case report. No Shinkei Geka 22: 677-680.

17. BAYRAKLI F, Sav A, Peker S (2010) Intracranial aneurysm mimicking cerebral cavernous malformation. J Nervous Sys Surg 3: 75-77.

18. Maenhoudt W, Hallaert G, Kalala J-P (2019) Complete resection of an intradural extramedullary foramen magnum cavernous malformation. World Neurosurg 129: 200-201. 
19. Mocco J, Laufer I, Mack WJ, Winfree CJ, Libien J, et al. (2005) An extramedullary foramen magnum cavernous malformation presenting with acute subarachnoid hemorrhage: Case report and literature review. Neurosurgery 56: E410-E410.

20. Wolfe SQ, Manzano G, Langer DJ, Morcos JJ (2011) Cavernous malformation of the oculomotor nerve mimicking a partially thrombosed posterior communicating artery aneurysm: Report of two cases. Neurosurgery 69: E470-E474.

21. Uneda A, Yabuno S, Kanda T, Suzuki K, Hirashita K, et al. (2017) Cavernous angioma presenting with subarachnoid hemorrhage which was diffusely distributed in the basal cisterns and mimicked intracranial aneurysm rupture. Surg Neurol Int 8: 202

22. Krings T, Mayfrank L, Thron A (2011) Bleeding from a cavernous angioma mimicking rupture of a middle cerebral artery aneurysm. Neuroradiology 43: 985-989.

23. Rheinboldt M, Blase J (2011) Exophytic hypothalamic cavernous malformation mimicking an extra-axial suprasellar mass. Emerg Radiol 18: 363-367.

24. Rapacki TFX, Brantley MJ, Furlow JTW, Geyer CA, Toro VE, et al. (1990) Heterogeneity of cerebral cavernous hemangiomas diagnosed by MR imaging. J Comput Assist Tomogr 14: 18-25.

25. Raps EC, Rogers JD, Galetta SL, Solomon RA, Lennihan L, et al. (1993) The clinical spectrum of unruptured intracranial aneurysms. Arch Neurol 50: 265-268.

26. Moriarity JL, Clatterbuck RE, Rigamonti D (1999) The natural history of cavernous malformations. Neurosurg Clin 10: $411-417$

27. Oishi T, Sakai N, Sameshima T, Kawaji H, Namba H (2017) The efficacy of resection of an intradural extramedullary foramen magnum cavernous malformation presenting with repeated subarachnoid hemorrhage: A case report. J Med Case Rep 11: 63.

28. Deshmukh VR, Albuquerque FC, Zabramski JM, Spetzler RF (2003) Surgical management of cavernous malformations involving the cranial nerves. Neurosurgery 53: 352-357.

29. Del Curling O, Kelly DL, Elster AD, Craven TE (1991) An analysis of the natural history of cavernous angiomas. $J$ Neurosurg 75: 702-708.

30. Pinker K, Stavrou I, Knosp E, Trattnig S (2006) Are cerebral cavernomas truly nonenhancing lesions and thereby distinguishable from arteriovenous malformations?: MRI findings and histopathological correlation. Magn Reson Imaging 24: 631-637.

31. Voigt K, Yasargil MG (1976) Cerebral cavernous haemangiomas or cavernomas. Neurochirurgia [Stuttg]. 19: $59-68$.

32. Das KK, Singh G, Pandey S, Bhaisora KS, Jaiswal A, et al. (2018) Completely thrombosed giant intracranial aneurysm with spontaneous thrombosis of the parent artery: Is it Nature's divine intervention and a self-cure? World Neurosurg 118: 132-138.

33. Cantu C, Murillo-Bonilla L, Arauz A, Higuera J, Padilla J, et al. (2005) Predictive factors for intracerebral hemorrhage in patients with cavernous angiomas. Neurol Res 27: 314318.

34. Pozzati E, Acciarri N, Tognetti F, Marliani F, Giangaspero F (1996) Growth, subsequent bleeding, and de novo appearance of cerebral cavernous angiomas. Neurosurgery 38: $662-670$.

35. Cianfoni A, Pravata E, De Blasi R, Tschuor CS, Bonaldi G (2013) Clinical presentation of cerebral aneurysms. Eur J Radiol 82: 1618-1622. 